\title{
Control perspective on synchronization and the Takens-Aeyels-Sauer reconstruction theorem
}

\author{
Henri Huijberts* \\ Faculty of Mathematics and Computing Science, Eindhoven University of Technology, P.O. Box 513, \\ 5600 MB Eindhoven, The Netherlands \\ Torsten Lilge \\ Institut für Regelungstechnik, Universität Hannover, Appelstrasse 11, D-30167 Hannover, Germany \\ Henk Nijmeijer \\ Faculty of Mathematical Sciences, University of Twente, P.O. Box 217, 7500 AE Enschede, The Netherlands \\ and Faculty of Mechanical Engineering, Eindhoven University of Technology, P.O. Box 513, 5600 MB Eindhoven, The Netherlands
}

(Received 15 June 1998)

\begin{abstract}
A method, based on ideas from control theory, is described for the synchronization of discrete time transmitter and receiver dynamics. Conceptually, the methodology consists of constructing observer-receiver dynamics that exploit the drive signal and past values of the drive signal at each time instant. In this way, the method can be viewed as a dynamic reconstruction mechanism. [S1063-651X(99)04904-1]

PACS number(s): 05.45.-a, 07.05.Dz, 02.10.Jf
\end{abstract}

\section{INTRODUCTION}

Following Pecora and Carroll [1], a great deal of interest in the synchronization of two coupled systems has arisen. This research is partly motivated by its possible use in secure communications, cf. Ref. [2]. Often, as in Ref. [1], a drive and response, or transmitter and receiver, viewpoint is assumed. In a discrete-time context, this typically allows for a description of the transmitter as an $n$-dimensional dynamical system,

$$
\begin{aligned}
& x_{1}(k+1)=f_{1}\left(x_{1}(k), x_{2}(k)\right), \\
& x_{2}(k+1)=f_{2}\left(x_{1}(k), x_{2}(k)\right),
\end{aligned}
$$

where $x_{1}(\cdot)$ and $x_{2}(\cdot)$ are vectors of dimensions $m$ and $l$, with $m+l=n$ and $x(k)=\left(x_{1}(k), x_{2}(k)\right)$. Given $x_{1}(\cdot)$ as the drive signal, the receiver dynamics are taken as a copy of Eq. (2):

$$
\tilde{x}_{2}(k+1)=f_{2}\left(x_{1}(k), \tilde{x}_{2}(k)\right) \text {. }
$$

Synchronization of the transmitter and receiver now corresponds to the asymptotic matching of Eqs. (2) and (3), that is,

$$
\lim _{k \rightarrow \infty}\left\|x_{2}(k)-\tilde{x}_{2}(k)\right\|=0
$$

Clearly Eq. (4) will not be satisfied in general and, in fact, conditions on $f_{1}$ and $f_{2}$ that guarantee this condition are only partially known, cf. Ref. [3]. For that reason several methods for achieving synchronization of signals like $x_{2}(\cdot)$ and $\tilde{x}_{2}(\cdot)$ have been proposed. In particular, we wish to recall the (reduced) observer viewpoint advocated in Ref. [4], which basically admits the construction of dynamics

\footnotetext{
*Author to whom correspondence should be addressed.
}

$$
\tilde{x}_{2}(k+1)=\tilde{f}_{2}\left(x_{1}(k), \tilde{x}_{2}(k)\right)
$$

such that Eq. (4) holds, whatever initial conditions Eqs. (1), (2), and (5) have. Although Eq. (5) supports the idea of using the copy [Eq. (3)] for Eq. (2), there are many systems for which Eq. (4) will not be met, no matter how $\widetilde{f}_{2}$ in Eq. (5) is chosen.

There is, however, a natural generalization of Eq. (5) that consists of exploiting the drive signal $x_{1}(k)$ and $x_{1}(k-1), \ldots, x_{1}(k-N)$ at each time instant $k$. Thus, as receiver dynamics, we use the following system:

$$
\widetilde{x}(k+1)=\widetilde{f}\left(\widetilde{x}(k), x_{1}(k), \ldots, x_{1}(k-N)\right) .
$$

Here $\widetilde{x}(\cdot)$ is $n$ dimensional, and $\widetilde{f}(\cdot, \cdot)$ and $N$ are such that

$$
\lim _{k \rightarrow \infty}|| x(k)-\widetilde{x}(k) \mid=0 .
$$

The receiver [Eq. (6)] acts as an "extended" observer for the system of equations (1) and (2) in that past values of the drive signal $x_{1}(\cdot)$ are also used. It turns out that under fairly weak conditions receiver dynamics [Eq. (6)] exist such that the transmitter [Eqs. (1) and (2)] and Eq. (6) synchronize; see Sec. II. Actually, the necessary conditions involved are closely related to global observability, cf. Ref. [5] or the Takens-Aeyels-Sauer reconstruction theorem (see Refs. $[6-9,3])$. However, a crucial difference in our work with the reconstruction theorem is that Eq. (6) forms a dynamic "inversion" for the state $x(\cdot)$, whereas in the reconstruction theorem one computes the state at some time instant by inverting the observability map, which determines $x_{2}(k)$ from $x_{1}(k), \ldots, x_{1}(k-N)$. It is interesting to note that an alternative using look-up tables for this procedure was proposed in Ref. [10].

The proposed transmitter and receiver synchronization using a receiver of form (6) can be demonstrated numerically on several examples from the literature; see, e.g., 
Refs. [11,12]. In this paper, we will consider, among others, the example from Ref. [11]. The organization of this paper is as follows. In Sec. II we present a design procedure for observer dynamics [Eq. (6)], where $N=n-1$. Section III presents numerical simulations of some synchronization problems where an observer presented in Sec. II is used. The paper ends with some concluding remarks.

\section{OBSERVER DESIGN}

In this section, we focus on an observer design for nonlinear, discrete-time, autonomous, single output systems of the forms

$$
x(k+1)=f(x(k)), \quad y(k)=h(x(k))
$$

for $k=0,1,2, \ldots$, where $x(\cdot)$ is a vector of dimension $n$ and $y(\cdot)$ is a scalar. Assuming that the Jacobian of $h$ is nonzero-which implies that a nontrivial signal from the dynamics is transmitted-we can, at least locally, rewrite Eq. (8) in a form like Eqs. (1) and (2), with $y(k)=x_{1}(k)$ being one dimensional. Within the context of synchronization, it is desired to reconstruct (asymptotically) the $(n-1)$-dimensional $x_{2}(\cdot)$ on the basis of the sequence $x_{1}(k)$ $(k=1,2, \ldots)$. We will do this using a suitably selected dynamics of form (6), which basically means that we treat the synchronization problem as a sort of observer problem; cf. Ref. [4]. Without loss of generality we can assume that $f(0)=0$ and $h(0)=0$. by

For Eq. (8) we define the so-called observability map $\psi$

$$
\psi(x):=\left[\begin{array}{c}
h(x) \\
h \circ f(x) \\
\vdots \\
h \circ f^{n-1}(x)
\end{array}\right],
$$

where $h \circ f(x):=h(f(x)), f^{1}:=f$, and $f^{j}:=f \circ f^{j-1}$. System (8) is called strongly locally observable around $x=0$ if the Jacobian $(\partial \psi / \partial x)(0)$ is invertible.

We now sketch a procedure to derive two different types of observers for the strongly locally observable system (8). This procedure was proposed in Refs. $[13,14]$, and represents an extension of Refs. $[15,16]$. For clarity of presentation, we will restrict ourselves to the case in which $n=3$. Extensions to other cases are straightforward.

Thus we consider a strongly locally observable system (8) with $n=3$, and define $s_{i}(x):=h \circ f^{i-1}(x)(i=1,2$, and 3). Since Eq. (8) is strongly locally accessible, $s$ $=\operatorname{col}\left(s_{1}, s_{2}, s_{3}\right)$ forms a new set of coordinates for Eq. (8) around $x=0$. In what follows, we will assume throughout that $s$ forms a new set of coordinates globally, i.e., $\psi$ in Eq. (9) is a global diffeomorphism on $\mathbb{R}^{n}$. It is straightforwardly checked that in these new coordinates the system (8) takes the form

$$
s(k+1)=\left[\begin{array}{c}
s_{2}(k) \\
s_{3}(k) \\
f_{s}(s(k))
\end{array}\right], \quad y(k)=s_{1}(k),
$$

where $f_{s}(s):=h \circ f^{3}\left(\psi^{-1}(s)\right)$. Next, define

$$
\begin{gathered}
z_{3}(k):=s_{1}(k), \\
z_{2}(k):=s_{2}(k)-f_{s}\left(y(k-2), y(k-1), s_{1}(k)\right), \\
z_{3}(k):=s_{3}(k)-f_{s}\left(y(k-1), s_{1}(k), s_{2}(k)\right) .
\end{gathered}
$$

It then follows from Eqs. (10) and (11) that $z$ $=\operatorname{col}\left(z_{1}, z_{2}, z_{3}\right)$ satisfies

$$
\begin{gathered}
z(k+1)=\left[\begin{array}{ccc}
0 & 0 & 0 \\
1 & 0 & 0 \\
0 & 1 & 0
\end{array}\right] z(k)+\left[\begin{array}{l}
0 \\
0 \\
f_{s}(y(k-2), y(k-1), y(k))
\end{array}\right], \\
y(k)=z_{3}(k)
\end{gathered}
$$

[where the first matrix is $E$ and the second is $\Phi(y(k$ $-2), y(k-1), y(k))]$.

An observer of type 1 now has the form

$$
\begin{aligned}
& \hat{z}(k+1)= E \hat{z}(k)+\Phi(y(k-2), y(k-1), y(k)) \\
&+ {\left[\begin{array}{c}
q_{0} \\
q_{1} \\
q_{2}
\end{array}\right][y(k)-\hat{y}(k)], } \\
& \hat{y}(k)=\hat{z}_{3}(k), \quad k \geqslant 2,
\end{aligned}
$$

where $q_{0}, q_{1}$, and $q_{2}$ are still to be determined. Defining the error signal $e:=\hat{z}-z$, we obtain the error dynamics

$$
e(k+1)=\left[\begin{array}{lll}
0 & 0 & -q_{0} \\
1 & 0 & -q_{1} \\
0 & 1 & -q_{2}
\end{array}\right] e(k),
$$

where the matrix is represented by $A$. The characteristic polynomial $p_{A}(\lambda)$ of $A$ is given by $p_{A}(\lambda)=\lambda^{3}+q_{2} \lambda^{2}$ $+q_{1} \lambda+q_{0}$. Choosing $q_{0}, q_{1}$, and $q_{2}$ in such a way that all eigenvalues of $A$ are located within the unit circle, the observer error $e(k)$ vanishes for $k \rightarrow \infty$, and condition (7) is met. From this it follows that the dynamics (13) initialized at an arbitrary point $\hat{z}(0)$ will asymptotically (even exponentially) match the transmitter dynamics (12). Therefore, the receiver dynamics (13) which is fed with the buffered transmitted signal $(y(k-2), y(k-1), y(k))$, synchronizes with Eq. (12).

The derivation of an observer of type 2 starts from the observation that the solutions of Eq. (12) satisfy $z_{1}(k)$ $=z_{2}(k)=0$ for $k \geqslant 2$. This suggests that one should consider an observer of the form

$$
\begin{gathered}
\hat{z}(k+1)=\Phi(y(k-2), y(k-1), y(k))+\left[\begin{array}{l}
\lambda_{1} \hat{z}_{1}(k) \\
\lambda_{2} \hat{z}_{2}(k) \\
\lambda_{3}(\hat{y}(k)-y(k))
\end{array}\right], \\
\hat{y}(k)=\hat{z}_{3}(k), \quad k \geqslant 2 .
\end{gathered}
$$

Again defining the error signal $e:=\hat{z}-z$, we now obtain the error dynamics

$$
e(k+1)=\left[\begin{array}{ccc}
\lambda_{1} & 0 & 0 \\
0 & \lambda_{2} & 0 \\
0 & 0 & \lambda_{3}
\end{array}\right] e(\dot{k})
$$


for $k \geqslant 2$. The convergence rate of the $i$ th component can now be assigned by $\lambda_{i}$, without affecting the other components. As was the case with observer 1, here we again have that the receiver dynamics (15), which is fed with the buffered transmitted signal $(y(k-2), y(k-1), y(k))$, synchronizes with Eq. (12).

Comparing both observer types, we see that the convergence rate of each of the components of observer type 2 can be assigned independently, while this is not the case for observer type 1 . Thus observer type 2 will give a better transient behavior than observer type 1 . On the other hand, however, observer type 1 with properly chosen $q_{0}, q_{1}$, and $q_{2}$ is in general more robust to (measurement) noise than observer type 2 ; cf. Refs. [13,14].

\section{EXAMPLES}

As an example, consider the transmitter systems

$$
\begin{aligned}
& x_{1}(k+1)=\mu(1-\epsilon) x_{1}(k)\left[1-x_{1}(k)\right]+\epsilon x_{2}(k), \\
& x_{2}(k+1)=\mu(1-\epsilon) x_{2}(k)\left[1-x_{2}(k)\right]+\epsilon x_{1}(k)
\end{aligned}
$$

presented in Ref. [11]. Taking $x_{1}(k)$ as the drive signal ( $m$ $=l=1$ ), Badola, Tambe, and Kulkarni investigated the synchronization of $x_{2}(k)$ and the receiver signal $x_{3}(k)$ of which the dynamics were taken as

$$
x_{3}(k+1)=\mu(1-\epsilon) x_{3}(k)\left[1-x_{3}(k)\right]+\epsilon x_{1}(k) .
$$

[In Ref. [11], $x_{2}(k)$ is considered as the drive signal. Since the coupled system given by Eq. (17) is symmetric, we can exchange $x_{1}(k)$ and $x_{2}(k)$ ]. Our aim is to apply an observer presented in Sec. II as the receiver dynamics for transmitter (17). With $y(k)=x_{1}(k)$, it is possible to design observers as in Sec. II in order to obtain the estimates $\hat{x}_{1}(k)$ and $\hat{x}_{2}(k)$ for the signals $x_{1}(k)$ and $x_{2}(k)$. The resulting observer equations are omitted for reasons of space. For subsequent simulations, the initial conditions $x_{1}(0)=0.2, x_{2}(0)=0.4$, and $\hat{x}_{1}(0)=\hat{x}_{2}(0)=0.7$ and parameters $\mu=3.7$ and $\epsilon=0.09$ were used. Following Ref. [11], $x_{2}(k)$ and $x_{3}(k)$ do not synchronize for these parameters and $x_{3}(0)=\hat{x}_{2}(0)=0.7$, while the observers obtained here show satisfactory behavior. Exemplary simulations of the observer errors applying observer types 1 and 2 can be seen in Figs. 1 and 2 for $\lambda_{1}=\lambda_{2}=0.5$ (for observer type 1 , this corresponds to the choices $q_{0}$ $=0.25$ and $\left.q_{1}=-1\right)$. Both observers provide very good estimations after 20 iterations with a maximum absolute observer error less than 0.002. As already mentioned in Sec. II, observer type 2 shows smaller observer errors during transient time than observer type 1 .

As a second example, we want to extend system (17) to the third order transmitter system

$$
\begin{aligned}
& x_{1}(k+1)=\mu(1-\epsilon) x_{1}(k)\left[1-x_{1}(k)\right]+\epsilon x_{2}(k), \\
& x_{2}(k+1)=\mu(1-\epsilon) x_{2}(k)\left[1-x_{2}(k)\right]+\epsilon x_{3}(k), \\
& x_{3}(k+1)=\mu(1-\epsilon) x_{3}(k)\left[1-x_{3}(k)\right]+\epsilon x_{1}(k),
\end{aligned}
$$

with the drive signal $y(k)=x_{1}(k)(m=1, l=2)$. In this case, observing the unknown signals $x_{2}(k)$ and $x_{3}(k)$ is more difficult because $x_{3}(k)$ does not directly influence the measured

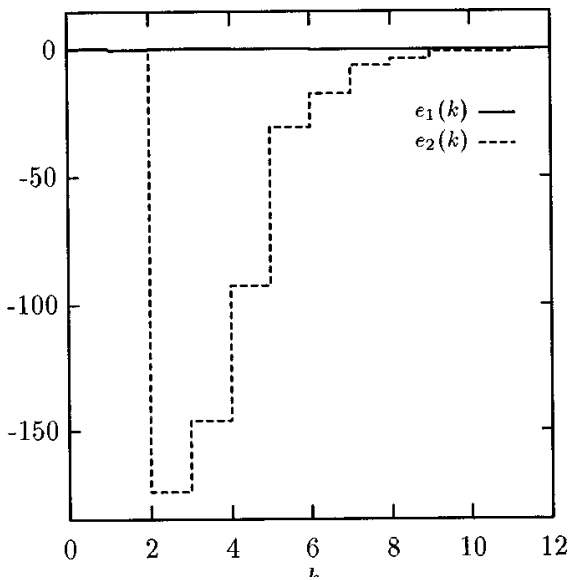

FIG. 1. Observer errors $e_{i}(k)=\hat{x}_{i}(k)-x_{i}(k)(i=1$ and 2) for system (17) and observer type 1 [Eq. (13)].

drive signal $x_{1}(k)$, but only via $x_{2}(k)$. For this reason, the coupling parameter $\epsilon$ was increased up to 0.35 while the second parameter $\mu=3.7$ was not changed. For $x_{1}(0)=0.2$, $x_{2}(0)=0.4, x_{3}(0)=0.6$, and $\hat{x}_{i}(0)=0.7, i=1,2$, and 3 , and eigenvalues of the observer error dynamics $\lambda_{i}=0.5, i=1,2$, and 3 (for observer type 1, this corresponds to the choices $q_{0}=-0.125, q_{1}=0.75$, and $\left.q_{2}=-1.5\right)$, the observer errors applying observer types 1 and 2 are shown in Figs. 3 and 4. It can be seen that $\left|e_{3}(k)\right|$ reaches very high values (up to 7500 with observer type 1) during the transient time. Nevertheless, after 20 iterations the maximum absolute observer error is less than 0.007 .

The examples show the efficiency of observers taken as receiver dynamics in synchronization problems, especially when taking into consideration that synchronization of the transmitter system and observer is guaranteed if the system is globally observable. Moreover, the eigenvalues of the observer error dynamics, and consequently the convergence rate, are selectable. For synchronization as presented in Ref. [11], one is neither able to guarantee synchronization nor able to influence the number of steps until synchronizations occurs.

\section{CONCLUDING REMARKS}

We have presented a control perspective on synchronization of discrete-time transmitter systems. The methodology

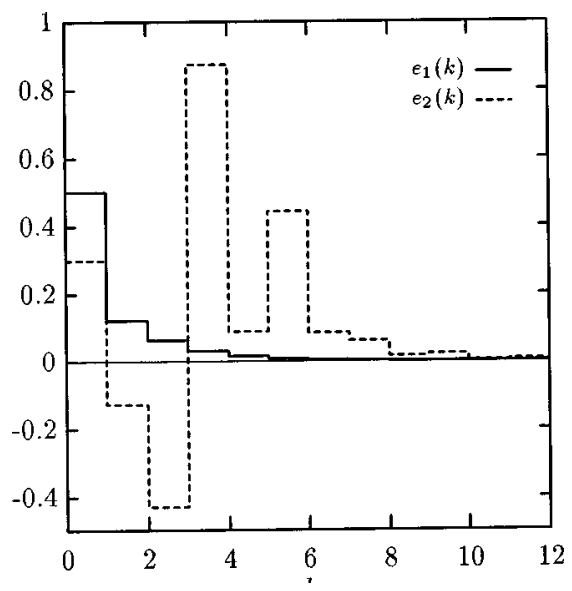

FIG. 2. Observer errors $e_{i}(k)=\hat{x}_{i}(k)-x_{i}(k)$ ( $i=1$ and 2) for system (17) and observer type 2 [Eq. (15)]. 


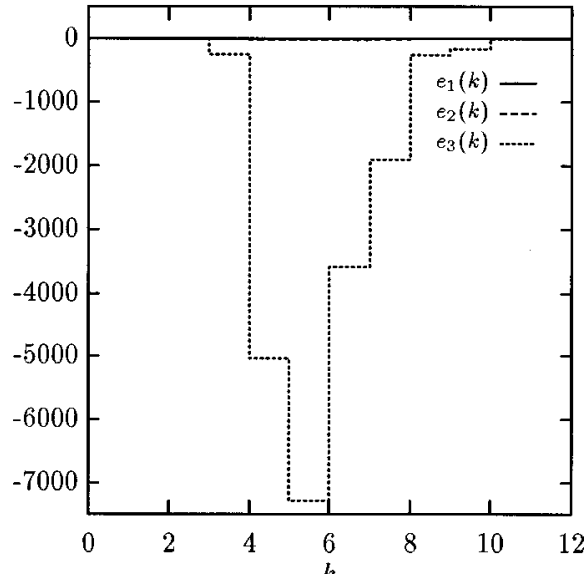

FIG. 3. Observer errors $e_{i}(k)=\hat{x}_{i}(k)-x_{i}(k)(i=1,2$, and 3) for system (19) and observer type 1 [Eq. (13)].

of designing an observer as the receiver system enables the exponential synchronization of the transmitter and receiver, and does not require any condition on conditional Lyapunov exponents, as is often the case when identical transmitter and receiver systems are used. Essentially, the observer scheme that is used in this paper exploits the last $n-1$ measurements of the drive signal $y(k), y(k-1), \ldots, y(k-n+1)$ at each time instant $k$, with $n$ being the dimension of the transmitter dynamics, and can be viewed as a dynamic mechanism for the (Takens-Aeyels-Sauer) reconstruction theorem, provided the system satisfies a global observability condition. Con-

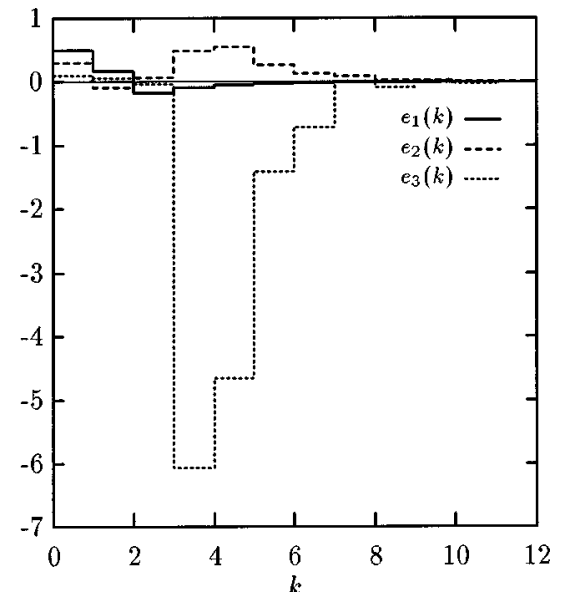

FIG. 4. Observer errors $e_{i}(k)=\hat{x}_{i}(k)-x_{i}(k)(i=1,2$, and 3$)$ for system (19) and observer type 2 [Eq. (15)].

trary to Ref. [11], our results are valid no matter how the initial conditions are chosen.

The observer viewpoint on the synchronization problem has also been advocated for continuous time systems (see Ref. [4]), but the scheme we used here in discrete time has no direct analog in continuous time. An obvious way to proceed in continuous time, therefore, could exist in a (fast) sampling of the continuous time transmitter and then the design of a discrete-time observer as receiver. In that case the synchronization error becomes small-depending on the sampling time-but not identically zero. However, in many applications this will not be a big problem.
[1] L. M. Pecora and T. L. Carroll, Phys. Rev. Lett. 69, 821 (1990).

[2] K. M. Cuomo and A. V. Oppenheim, Phys. Rev. Lett. 71, 65 (1993).

[3] Coping with Chaos, edited by E. Ott, T. Sauer, and J. A. Yorke (Wiley, New York, 1994).

[4] H. Nijmeijer and I. M. Y. Mareels, IEEE Trans. Circuits Syst., I: Fundam. Theory Appl. 44, 882 (1997).

[5] H. Nijmeijer, Int. J. Control 36, 867 (1982).

[6] F. Takens, in Dynamical Systems and Turbulence, edited by D. A. Rand and L. S. Young (Springer-Verlag, Berlin, 1981).

[7] D. Aeyels, SIAM J. Control Optim. 19, 595 (1981).

[8] D. Aeyels, Syst. Control Lett. 1, 92 (1981).

[9] T. Sauer, in Time Series Prediction: Forecasting the Future and Understanding the Past, edited by A. S. Weigend and N. A. Gershenfield (Addison-Wesley, Reading, MA, 1993).

[10] P. E. Moraal and J. W. Grizzle, IEEE Trans. Autom. Control. 40, 395 (1995).

[11] P. Badola, S. S. Tamble, and B. D. Kulkarni, Phys. Rev. A 46, 6735 (1992).

[12] M. Loecher, and E. R. Hunt, Phys. Rev. Lett. 79, 63 (1997).

[13] T. Lilge, Eur. J. Control 4, 306 (1998).

[14] T. Lilge, in Proceedings of the 1997 COSY Workshop on Control of Nonlinear and Uncertain Systems, edited by A. Isidori and F. Allgöwer (ETH, Zürich, 1997), p. 202.

[15] M. Brodmann, Beobachterentwurf für Nichtlineare Zeitdiskrete Systeme (VDI-Verlag, Düsseldorf, 1994).

[16] W. Lin and C. I. Byrnes, Syst. Control Lett. 25, 31 (1995). 